\title{
Experimental Investigations of 3D Woven Layer to-Layer Carbon/Epoxy Composites at Different Strain Rates
}

\author{
Sanghyun Yoo $^{1 *}$, Monali Dahale ${ }^{2}$, Geoffrey Neale $^{2}$, Nathalie Toso ${ }^{1}$, Giuseppe Catalanotti ${ }^{3}$, \\ Alistair McIlhagger ${ }^{2}$, Edward Archer $^{2}$, Eileen Harkin-Jones ${ }^{2}$, and Heinz Voggenreiter ${ }^{1}$ \\ ${ }^{1}$ Institute of Structures and Design, German Aerospace Center (DLR), Stuttgart, Germany \\ ${ }^{2}$ Engineering Research Institute, Ulster University, Newtownabbey, UK \\ ${ }^{3}$ Advanced Composites Research Group, School of Mechanical and Aerospace Engineering, Queen's \\ University Belfast, Belfast, UK
}

\begin{abstract}
This paper reports experimental investigations of 3D woven carbon/epoxy composites on quasi-static and dynamic tensile properties in the longitudinal (warp) and transverse (weft) directions. Firstly, quasi-static tests were conducted to determine a baseline tensile strength and to find out the adequate specimen geometry required for dynamic testing. Secondly, dynamic tensile properties at intermediate strain rates (nominal strain rates from 0.1 to $200 \mathrm{~s}^{-1}$ ) were investigated alongside the corresponding failure mechanisms. Detailed information on failure patterns is obtained with strain field measurements from Digital Image Correlation (DIC) and CT scans. The results show that 3D woven composites are strain rate insensitive and the crack initiation is located near weft yarns and binding interlacement points due to the presence of resin rich areas.
\end{abstract}

\section{Introduction}

There is the tremendous interest in using lightweight composite materials for load bearing structures in transportation applications. This is driven by the need for a reduction in energy consumption and subsequent compliance with environmental regulations. The design of transportation structures using composites instead of metallic materials may help to reduce energy consumption by decreasing structural weight. To make such a transition from metallic to composites, it is necessary to ensure the ability of newly introduced composite structures to meet current safety legislation and certification processes on crashworthiness. Therefore, knowledge about the dynamic properties at intermediate strain rates $\left(1-200 \mathrm{~s}^{-1}\right)$ is particularly important to understand behaviour during automotive crash events [1].

Dynamic testing at intermediate strain rates is inherently difficult due to intrinsic problems associated with inertia effects and stress wave propagation. Neither the traditional quasi-static approach nor the classical Split-Hopkinson Tensile Bar (SHTB) techniques are suitable to acquire dynamic material properties at intermediate strain rates [2]. The inertial

* Corresponding author: Sanghyun.Yoo@dlr.de 
effect, known as system ring, is one of the significant obstacles to obtaining true dynamic properties at intermediate strain rates. When the load is introduced to the specimen, high amplitude stress waves are generated in the test machine and test specimen resulting in high oscillations in force measurement at the natural frequency of the system. Those undesirable oscillations complicate the interpretation of force measurement [3].

3D fibre reinforced composites are known to offer several advantages for transportation structures over traditional 2D laminate composites in terms of delamination resistance, damage tolerance and design flexibility [4-6]. However, there is a lack of available literature on rate dependent properties of 3D woven composites due to experimental challenges, especially on specimen size [7]. Therefore, there is a need to investigate dynamic test procedures that can obtain reliable and accurate strain rate dependent properties for $3 \mathrm{D}$ woven composites. In this study, we investigate quasi-static and dynamic tensile properties in the longitudinal (warp) and transverse (weft) directions of 3D woven carbon/epoxy composites tested at nominal strain rates up to $200 \mathrm{~s}^{-1}$. The main aim of this work is to investigate experimental techniques for strain rate dependent properties of $3 \mathrm{D}$ woven composites. A unique testing approach, involving adhesive clamping methods and DIC techniques, is used to overcome aforementioned testing challenges. Also, CT scans are used to understand the failure mechanisms of this 3D woven architecture. This study will be the first step towards exploring the influence of specimen size on dynamic testing of 3D woven composites and will lead to the development of a reliable dynamic material characterization technique.

\section{Materials and Methods}

\subsection{Materials}

Fig. 1 (a) shows the textile architecture of a 3D woven layer-to-layer architecture generated using ScotWeave design software. The chosen architecture consists of a weft density of 10 wefts $/ \mathrm{cm}$ and a warp density of 12 warps $/ \mathrm{cm}$. The $3 \mathrm{D}$ woven preforms were constructed with T700S-50C-12k carbon fibres from Toray Industries, Inc.
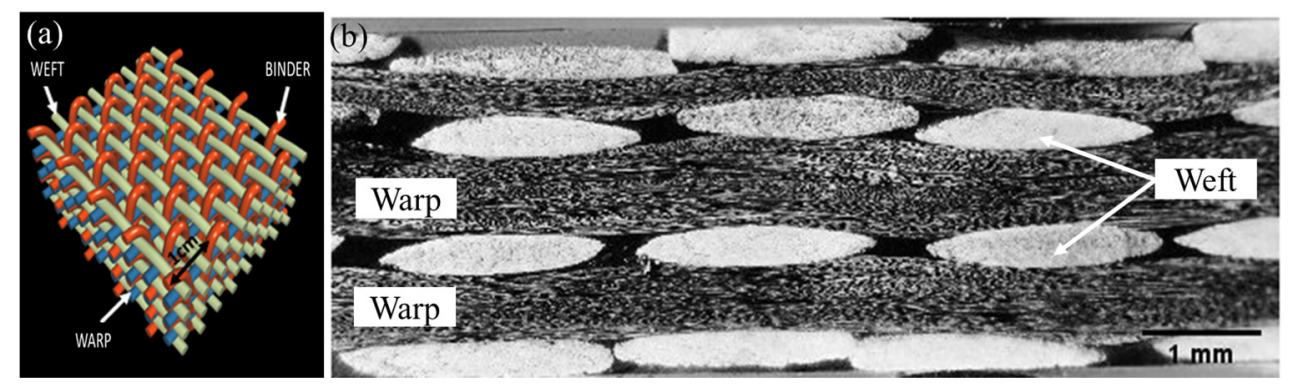

Fig. 1. 3D woven layer-to-layer architecture (a) A textile design pattern (red: binder yarns, Blue: warp yarns, light green: weft yarns) and (b) A microscopy image of warp direction cross-section.

The unit cell size of repeating geometry from a dry preform was found to be $12 \mathrm{~mm}$ in both the warp and weft directions. The preform was infused by Resin Transfer Moulding (RTM) with epoxy resin (PRIMETM 20LV from Gurit) and cured at $50{ }^{\circ} \mathrm{C}$ for 16 hours at 1 bar pressure. Fig. 1 (b) shows a microscopic image for an internal structure in the warp 
direction, and the summary of preform properties is reported in Table 1 . The details of the $3 \mathrm{D}$ woven layer-to-layer architectures can be found in [8].

Table 1. Summary of the preform properties of 3D woven layer-to-layer architecture.

\begin{tabular}{|c|c|c|c|c|c|c|}
\hline \multirow{2}{*}{$\begin{array}{c}\text { Areal } \\
\text { Density } \\
{[\mathrm{kg} / \mathrm{m} 2]}\end{array}$} & \multicolumn{3}{|c|}{$\begin{array}{c}\text { Yarn content } \\
{[\%]}\end{array}$} & \multicolumn{2}{|c|}{ \% Tow Crimp } & \multirow{2}{*}{$\begin{array}{c}\begin{array}{c}\text { Unit cell size } \\
\text { [mm] }\end{array} \\
\text { Warp/Weft }\end{array}$} \\
\hline & Warp & Weft & Binder & Warp & Weft & \\
\hline 1.45 & 27.2 & 45.5 & 27.2 & 3.7 & 1.4 & $12 / 12$ \\
\hline
\end{tabular}

Fig. 2 (a) shows a schematic of the specimen geometry used for quasi-static testing. Specimens, with a nominal length and width of $165 \mathrm{~mm}$ and $10 \mathrm{~mm}$ respectively, were cut with a water-cooled diamond saw. Tabs made of glass fabric/epoxy laminates cut in the $45^{\circ}$ direction were bonded to facilitate smooth load introduction into the test specimen without initiating premature failure. Fig. 2 (b) shows the dynamic tension specimens which were cut in a dog-bone shape with a gauge length of $10 \mathrm{~mm}$ to encourage specimen failure at the central gauge section.

(a)

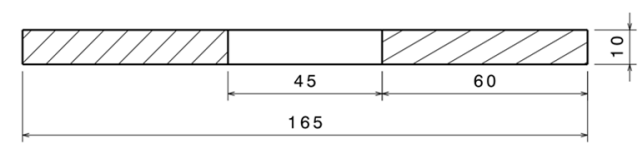

(b)

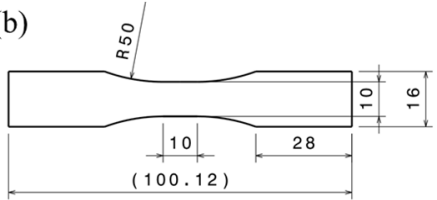

Fig. 2. Schematic of specimen geometries for tensile testing at (a) quasi-static condition, and (b) intermediates strain rates (Unit is in $\mathrm{mm}$ ).

The dynamic impulse from load introduction can excite the testing system at its natural frequency. This effect results in oscillations in the load measurement that obscure the interpretation of the test data. To reduce the system ringing, the grips can be modified to increase the natural frequency of the system through lightweight design concepts. To increase the natural frequency of the test system, a lightweight clamping method was used which involves using slotted grips for adhesive bonding. These slotted grips had threaded end-caps that allowed connection of the specimens to the slack adapter. The clamping technique using adhesives was inspired by $[9,10]$, where it was used to connect the material specimen to SHTB. A two components epoxy (Scotch Weld DP490 from 3M ${ }^{\mathrm{TM}}$ ) was used to establish proper bonding between specimens and grips. After that, the bonded specimens were postcured in the furnace at $65^{\circ} \mathrm{C}$ for 2 hours to improve the performance of the bond (according to the manufacturer's recommendation). The selection of the geometry was based on quasistatic tensile strength of tested materials and shear strength of the adhesive.

\subsection{Test methods}

For the quasi-static loading case, tensile tests were carried out using a universal test machine, Zwick/Roell 1484 equipped with a $200 \mathrm{kN}$ load cell and hydraulic specimen grips. Rectangular specimens were used to carry out the testing with a displacement control of 2 $\mathrm{mm} / \mathrm{min}$ and a clamping pressure of 200 bars. A 2-element rosette strain gauge (a gauge length of $3 \mathrm{~mm}$ ), FCA-3-11-1L from Tokyo Sokki Kenkyujo Co., Ltd. was attached to the 
centre of the specimen on the backside to measure the strain. Surface strain was acquired using digital image correlation (DIC) with a Cannon EOS 60D CCD camera. Images were then processed with $\mathrm{GOM}^{\circledR}$ Correlate software. The resolution of the CCD camera was 1088 x 1920 pixels, and the frame rate was 25 frames per second. The surface field was created with a facet size of 10 pixels and a point distance of 10 pixels. The strain was calculated from the strain field and used to establish stress-strain data. Five specimens were tested.

Dynamic tensile tests at nominal strain rates from 0.1 to $100 \mathrm{~s}^{-1}$ were carried out with a high-speed servo hydraulic testing machine of type Instron ${ }^{\circledR}$ VHS 100/20. A piezo-electric load cell (Kistler-9317B, Kistler Instrumente $\mathrm{GmbH}$ ) was used to measure the force, which was then amplified with a Kistler type 5011B charge amplifier. The strain and strain rates were obtained from the DIC technique. The DIC images were captured with a high-speed camera (FASTCAM SA-Z, Photron ${ }^{\circledR}$ ) and were then processed with GOM $^{\circledR}$ Correlate software. The processing parameters were chosen identical to the quasi-static cases to minimise variation in results. The resolution was varied with respect to the frame rate to ensure the number of output data points (i.e., the resolution of $384 \times 216$ pixels with 160,000 frame rates was used at nominal strain rate of $200 \mathrm{~s}^{-1}$ ). In addition, high-speed images were synchronised with force signals thorough NI-DAQ (USB-6251 BNC, National Instruments $\left.^{\mathrm{TM}}\right)$. Furthermore, it is worth mentioning that no filtering was applied to the measured stress-strain data, and that three specimens were tested at each strain rate.

It should be noted here that the strain gauge is only sufficient for local spatial resolution in the woven material. The textile repeat pattern is $12 \mathrm{~mm}$ (refer to Table 2) but the strain gauge length is $3 \mathrm{~mm}$ so the cell size is much greater than the strain gauge dimension. For that reason, the DIC technique, which is a full-field optical measurement, is used here in addition to the strain gauge. Limitations of using strain gauges with $3 \mathrm{D}$ woven composites have also been described in $[4,5,11]$, where the authors reached the conclusion that the DIC method is preferred over strain gauges.

\section{Results and Discussion}

\subsection{Quasi-static tension properties}

Since a smaller specimen geometry is generally preferred for dynamic testing due to the concerns with dynamic stress equilibrium and system ringing [1], it was necessary to consider a specimen geometry for quasi-static tests, which is close to the specimen geometry used for dynamic testing. Fig. 3 shows quasi-static transverse tensile results of 3D woven composites from a universal testing machine (Zwick) and a high-speed servo-hydraulic machine (VHS). Detailed quasi-static mechanical properties are reported in Table 2.

Experimental results from testing at strain rates of $0.1 \mathrm{~s}^{-1}$ and $4 \cdot 10^{-4} \mathrm{~s}^{-1}$ are shown in fig. 3 and table 2 and show no significant difference between test speeds (both tests are considered to be in the quasi-static regime which is defined as being from $10^{-4} \mathrm{~s}^{-1}$ to $10^{-1} \mathrm{~s}^{-1}$ according to [12]). 


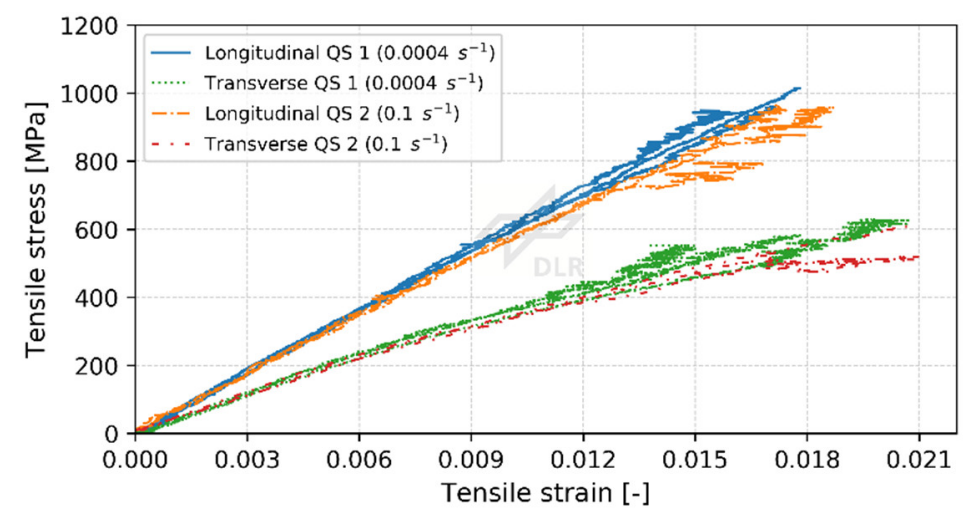

Fig. 3. Measured quasi-static stress-strain data of 3D woven composites for longitudinal and transverse tension properties using different testing machines.

Table 2. Summary of tension properties of 3D woven composites in longitudinal and transverse directions under quasi-static testing condition gained from different testing machines.

\begin{tabular}{ccccc}
\hline Direction & $\begin{array}{c}\text { Strain rates } \\
{\left[\mathbf{s}^{-1}\right]}\end{array}$ & $\begin{array}{c}\text { Young's modulus } \\
{[\mathbf{G P a}]}\end{array}$ & $\begin{array}{c}\text { Tensile strength } \\
{[\mathbf{M P a}]}\end{array}$ & $\begin{array}{c}\text { Failure strain } \\
{[-]}\end{array}$ \\
\hline \multirow{2}{*}{ Longitudinal } & $0.0004(\mathrm{QS} 1)$ & $60.8 \pm 5.0$ & $958.00 \pm 54.53$ & $0.017 \pm 0.001$ \\
& $0.1(\mathrm{QS} 2)$ & $56.7 \pm 2.7$ & $923.94 \pm 38.33$ & $0.018 \pm 0.001$ \\
\hline \multirow{2}{*}{ Transverse } & $0.0004(\mathrm{QS} 1)$ & $39.4 \pm 3.5$ & $583.59 \pm 28.17$ & $0.018 \pm 0.002$ \\
& $0.1(\mathrm{QS} 2)$ & $37.0 \pm 2.0$ & $559.61 \pm 39.85$ & $0.019 \pm 0.002$ \\
\hline
\end{tabular}

\subsection{Dynamic tension properties}

Fig. 4 and Fig. 5 depict the dynamic tensile properties of 3D woven composites in the longitudinal and transverse direction, respectively at nominal strain rates up to $200 \mathrm{~s}^{-1}$. The materials exhibit almost linear strain-stress relationships in both directions. A comparison of all results is shown in Fig. 6. The experimental results indicate that the current layer-to-layer $3 \mathrm{D}$ architecture is insensitive to strain rate variation. This is coherent with the fact that longitudinal carbon fibre shows no strain-rate sensitivity [13, 14]. Also, Tran. et al. [15] reported strain-rate insensitivity of 3D carbon/epoxy composites in a similar strain rate range. These results show that the proposed test procedures using the developed slack adapter and an adhesive bond clamping method can generate 'clean' dynamic mechanical test data for $3 \mathrm{D}$ woven composites. 


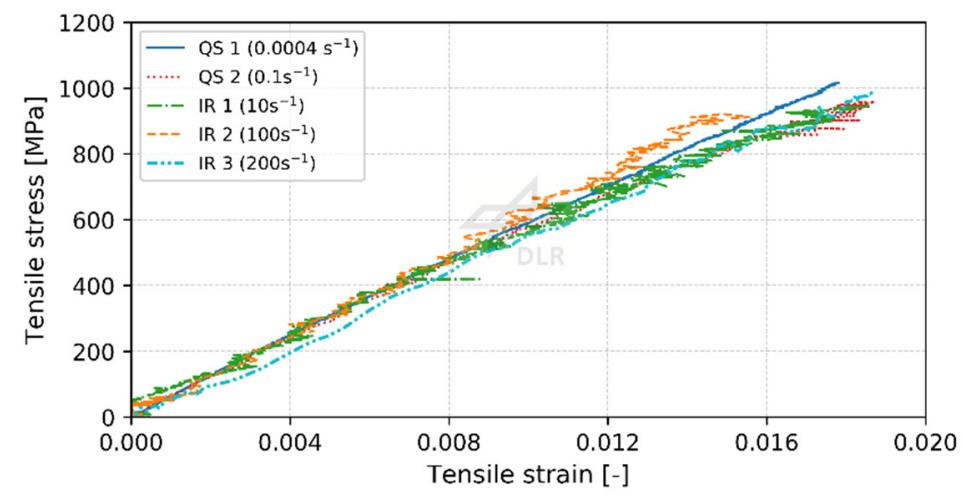

Fig. 4. Measured stress-strain data of $3 \mathrm{D}$ woven composites in longitudinal (warp) direction at different strain rates from quasi-static to $200 \mathrm{~s}^{-1}$.

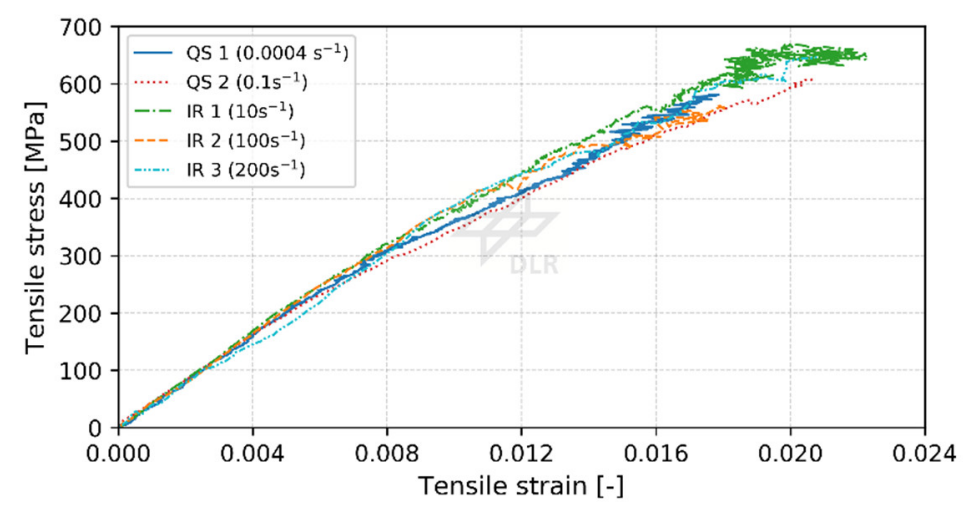

Fig. 5. Measured stress-strain data of $3 \mathrm{D}$ woven composites in transverse (weft) direction at different strain rates from quasi-static to $200 \mathrm{~s}^{-1}$.

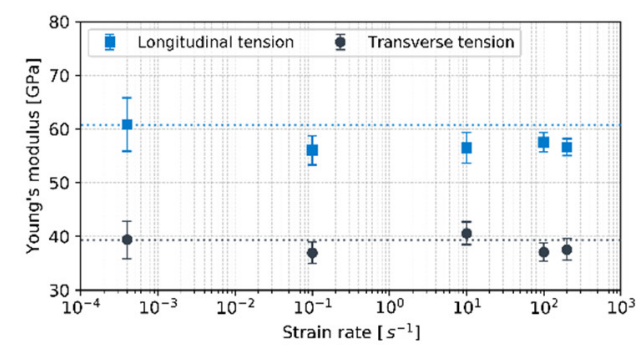

(a)

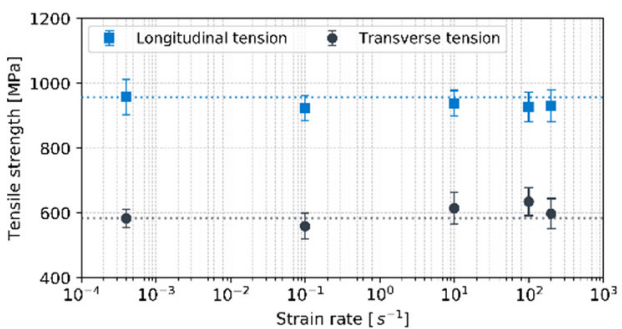

(b)

Fig. 6. Strain rate dependent properties of 3D woven composites in longitudinal and transverse directions for (a) Young's modulus, and (b) tensile strength (dot lines indicate the quasi-static value as reference).

\subsection{Failure mechanism}

Fig. 7 (a) shows superimposed images of a pristine specimen in the longitudinal direction and the corresponding surface strain field obtained from DIC images. The images show that high strain values develop near binding points (weave crimping regions) and weft yarns (in red). Dahale. et al. [8] investigated quasi-static tension in a similar 3D layer-to-layer 
architecture in glass/epoxy composites and found that resin rich areas around weft yarns and binding interlacement points induced cracks as stress concentration in quasi-static tension testing. Thus, it can be said that surface cracks observed in DIC images are matrix cracking resulting from the resin rich areas around binder interlacement points where the binder changes the direction and within weft yarns.

(a)

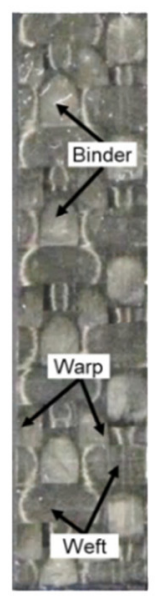

Pristine

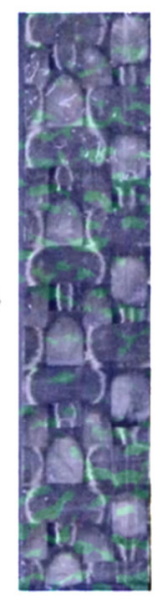

Strain at 0.003

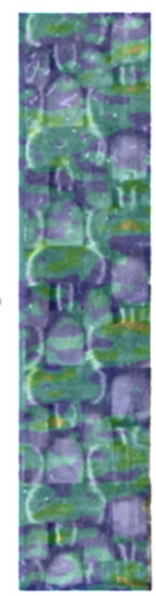

Strain at 0.009

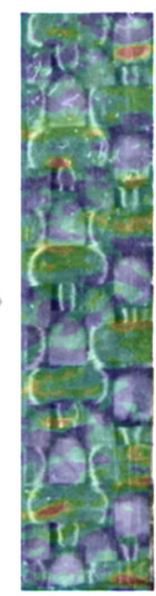

Strain at 0.012
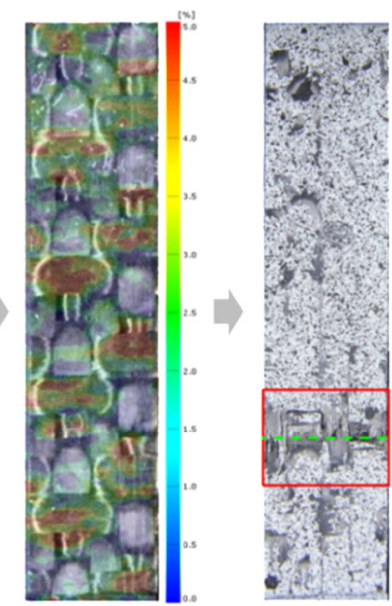

At failure

(b)
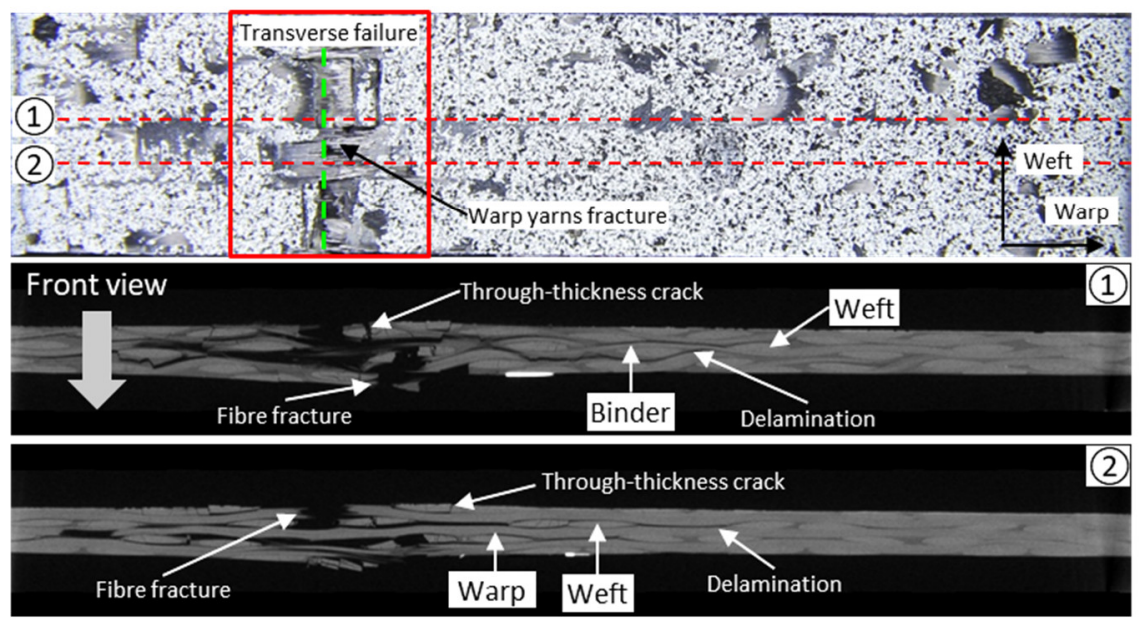

Fig. 7. Superimposed images of specimen surface and DIC images (strain values are referring to Fig. 4), and (b) surface image of failed specimen with corresponding CT images of 3D woven composites tested in longitudinal direction.

Fig. 7 (b) shows the surface image of the failed specimen tested in the longitudinal direction and its corresponding CT scans. The straight transverse failure and longitudinal fibre fractures are clearly observed. Based on experimental observation at the macroscale, the crack growth path can be explained as follow: (i) the matrix crack starts at the interlacement points between binder and weft yarns where a resin-rich area exists, which can be confirmed by Fig. 7 (a). (ii) the matrix cracks coalesce into a lager transverse crack and binder yarn straightening leads the delamination along the boundary of the binder yarns. (iii) the delamination leads to longitudinal fibre (warp yarns) breakage. Furthermore, consistent failure patterns (transverse failure) were observed in failed specimens from dynamic testing at intermediate strain rates. Those results can be explained by the fact that the failure mode 
in the longitudinal direction is fibre dominant, which is also related to almost identical stressstrain curves from quasi-static to nominal strain rates up to $200 \mathrm{~s}^{-1}$.

\section{Conclusions}

Comprehensive dynamic mechanical characterisation of 3D woven composites in the longitudinal and transverse directions was performed from quasi-static to strain rates up to $200 \mathrm{~s}^{-1}$. Results of the experimental investigation are: (i) the longitudinal tensile properties of the 3D architecture in the warp direction show a linear stress-strain relationship, and transverse tensile properties show a similar stress-strain relationship. (ii) strain rate insensitivity of the architecture, up to a nominal strain rate of $200 \mathrm{~s}^{-1}$.

It can be concluded that reliable and concise experimental data can be obtained using the proposed dog-bone specimen geometry $(100 \mathrm{~mm} \times 16 \mathrm{~mm})$. The test procedures in this work have allowed acquisition of reliable dynamic mechanical properties of 3D woven carbon/epoxy composites. In future work the key factors to determine optimal specimen geometry will be investigated with a concurrent consideration between obtaining a homogenous stress field via a small gauge section size and a sufficient covering of unit cell of the specific $3 \mathrm{D}$ architecture.

This work was supported by the EU Horizon 2020 Marie Skłodowska-Curie Actions Innovative Training Network ICONIC: Improving the crashworthiness of composite transportation structures [grant agreement No. 721256].

\section{References}

1. X. Xiao, Dynamic tensile testing of plastic materials, Polymer Testing 27, 164-178 (2008).

2. T.J. Cloete, M. Stander, Refinement of the wedge bar technique for compression tests at intermediate strain rates, EPJ Web of Conferences 26, 01025 (2012).

3. M.M. LeBlanc, D.H. Lassila, A hybrid technique for compression testing at intermediate strain rates, Experimental Techniques 20, 21-24 (1996).

4. S. Dai, P.R. Cunningham, S. Marshall, C. Silva, Influence of fibre architecture on the tensile, compressive and flexural behaviour of $3 D$ woven composites, Composites Part A: Applied Science and Manufacturing 69, 195-207 (2015).

5. J.P. Quinn, A.T. Mcllhagger, R. McIlhagger, Examination of the failure of $3 D$ woven composites, Composites Part A: Applied Science and Manufacturing 39, 273-283 (2008).

6. M.N. Saleh, C. Soutis, Recent advancements in mechanical characterisation of $3 D$ woven composites, Mechanics of Advanced Materials and Modern Processes 3, 12 (2017).

7. R. Gerlach, C.R. Siviour, J. Wiegand, N. Petrinic, In-plane and through-thickness properties, failure modes, damage and delamination in $3 D$ woven carbon fibre composites subjected to impact loading, Composites Science and Technology 72, 397411 (2012).

8. M. Dahale, G. Neale, R. Lupicini, L. Cascone, C. McGarrigle, J. Kelly, E. Archer, E. Harkin-Jones, A. McIlhagger, Effect of weave parameters on the mechanical properties of $3 D$ woven glass composites, Composite Structures 223, 110947 (2019). 
9. A. Gilat, R.K. Goldberg, G.D. Roberts, Experimental study of strain-rate-dependent behavior of carbon/epoxy composite, Composites Science and Technology 62, 14691476 (2002).

10. H. Koerber, P. Kuhn, M. Ploeckl, F. Otero, P.-W. Gerbaud, R. Rolfes, P.P. Camanho, Experimental characterization and constitutive modeling of the non-linear stressstrain behavior of unidirectional carbon-epoxy under high strain rate loading, Advanced Modeling and Simulation in Engineering Sciences 5, 17 (2018).

11. A. Doitrand, C. Fagiano, V. Chiaruttini, F.H. Leroy, A. Mavel, M. Hirsekorn, Experimental characterization and numerical modeling of damage at the mesoscopic scale of woven polymer matrix composites under quasi-static tensile loading, Composites Science and Technology 119, 1-11(2015).

12. C.R. Siviour, J.L. Jordan, High Strain Rate Mechanics of Polymers: A Review, Journal of Dynamic Behavior of Materials 2, 15-32 (2016).

13. J. Harding, L.M. Welsh, A tensile testing technique for fibre-reinforced composites at impact rates of strain, Journal of Materials Science 18, 1810-1826 (1983).

14. N. Taniguchi, T. Nishiwaki, H. Kawada, Tensile strength of unidirectional CFRP laminate under high strain rate, Advanced Composite Materials 16, 167-180 (2007).

15. N. Tran, J. Berthe, M. Brieu, G. Portemont, E. Deletombe, J. Schneider, Characterisation of high strain rate dependency of $3 D$ woven CFRP materials, in Proceedings of the 17th European Conference on Composite Materials (ECCM17), Munich, Germany (2016). 